\title{
Ueber die Bestandteile der Wurzel von Baptisia tinctoria.
}

Von Dr. K. Gorter.

(Fortsetzung.)

Erwärmte ich die Naphtalindoppelverbindung der durch Einwirkung von Salpetersäure auf Baptisin erhaltenen Styphninsäure gelinde mit etwas Wasser und filtrierte, dann gab das Filtrat nach Zusatz von Natronlauge und einigen Tropfen Jodjodkaliumlösung einen penetranten Jodoformgeruch.

Auch der Gewichtsverlust beim Trocknen bei $100^{\circ} \mathrm{C}$., wobei das Naphtalin und das Aceton entweichen, steht in bestem Einklang mit der Formel mit Krystallaceton.

$0,4630 \mathrm{~g}$ verloren beim Trocknen bei $100^{\circ} \mathrm{C} .0,2102 \mathrm{~g}$ an Gewicht. Gewichtsverlust.

Gefunden Berechnet für $\mathrm{C}^{6} \mathrm{H}^{3} \mathrm{~N}^{3} \mathrm{O}^{8} \cdot \mathrm{C}^{10} \mathrm{H}^{8}+\mathrm{C}^{3} \mathrm{H}^{6} \mathrm{O}$ $43,37 \%$

Alles oben mitgeteilte wäre schon ausreichend für die Beweis. fübrung der Identität meiner Substanz mit der Styphninsäure. Ich kann jedoch noch hinzufügen, dass das Kalisalz sich mit einem Ueber. schuss von Kalilauge als schwer lösliche orangerote Nädelchen ausfällen lässt. Auch das Baryt- und das Silbersalz sind schwer löslich. Auch das Verhalten bei der Reduktion ist ebenfalls dem der Styphninsäure gleich.

Durch die Bildung der Styphninsäure ist das Baptigenin als ein zur aromatischen Reihe gehöriger Körper, welcher einen Resorcinkern enthält, gekennzeichnet. Das zweite Einwirkungsprodukt der Salpetersäure, welches sich beim Verdünnen mit Wasser ausscheidet, habe ich trotz mehrfacher Bemühung noch nicht analysenrein erhalten können.

Kalischmelze.

Die Nitrierung des Baptisins lehrt, dass dieses Glykosid, bezw. Rhamnosid einen Resorcinkern enthält, da hierbei Styphninsäure (Trinitroresorcin) in beträchtlicher Menge entsteht. Es war daher bei der vorsichtigen Kalischmelze des Baptisins, bez. des Baptigenins, ebenfalls die Bildung von Resorein zu erwarten.

$10 \mathrm{~g}$ Aetzkali wurden zur Prüfung dieser Vermutung im Nickeltiegel in 2 cc Wasser gelöst und $1 \mathrm{~g}$ Baptisin eingetragen. Das Gemisch wurde auf kleiner Flamme erhitzt. Es trat hierbei starkes Aufschäumen und Entwicklung eines aromatischen Geruches ein. Es wurde hin und wieder die Flamme fortgenommen. Das Schmelzen

Arch. d. Pharm. CCXXXV. Bds. 5. Heft. 
wurde mit dieser Vorsicht während einer halben Stunde fortgesetzt. Die Masse hatte sich dann eidottergelb gefärbt; allein an der Oberfläche war sie etwas braun. Die Schmelze löste sich nach dem Abkühlen in verdünnter Schwefelsäure unter Kohlensäureentwicklung zu einer klaren Flüssigkeit mit kreosotartigem Geruch.

Diese Lösung wurde mit Aether ausgeschüttelt und dieser an der Luft verdunstet. Es resultierte hierbei eine geringe Menge weisser Nädelchen, welche durch Pressen zwischen Fliesspapier von der anhängenden, gelbroten Mutterlauge befreit wurden. Sie zeigten den Schmelzpunkt $96^{\circ}$ (unk.). Eisenchlorid gab mit der wässrigen Lösung eine Grünfärbung, welche sich auf Zusatz ron Sodalösung in Violett umwandelte.

Eine ammoniakalische Silberlösung (Silbernitratlösung mit einem Tropfen Natronlauge und Ammoniakflüssigkeit zur Lösung) wurde sofort reduziert. Der Körper war sublimierbar, kennzeichnete sich somit in seinen Eigenschaften als Brenzeatechin.

Resorcin konnte bei dieser kleinen Probe nicht nachgewiesen werden. Es wurde daher dieser Versuch mit einiger Abänderung in etwas grösserem Umfange wiederholt.

$10 \mathrm{~g}$ Kaliumhydroxyd wurden mit 2 ec Wasser im Nickeltiegel geschmolzen und $3 \mathrm{~g}$ Baptisin eingetragen. Es trat hierbei Aufschäumen und Entwickelung eines aromatischen Geruches auf. Das Schmelzen wurde auf kleiner Flamme so lange fortgesetzt, bis sich eine Probe, in Wasser gelöst, durch Zusatz von verdünnter Schwefelsäure nicht mehr trübte. Diese Operation dauerte 40 Minuten. Die Schmelze wurde dann in Wasser gelöst und weiter, wie in Tabelle Seite 323 angegeben, verarbeitet.

Es entstehen somit bei der Kalischmelze des Baptisins im Wesentlichen Brenzkatechin, Resorcin und Ameisensäure; nebenbei treten auch Spuren von Guajacol und anderer nicht näher definierter Substanzen auf.

Oxydation des Baptigenins mit übermangansaurem Kalium.

Es wurde jetzt versucht durch die Oxydation mit Kaliumpermanganat einen weiteren Einblick in die Struktur des Baptigenins zu gewinnen.

0,500 g Baptigenin wurden zu diesem Zwecke mit Wasser zerrieben und etwas verdünnte Schwefelsäure hinzugesetzt. $\mathrm{Zu}$ dieser Flüssigkeit fügte ich in kleinen Mengen bei gewöhnlicher Temperatur 


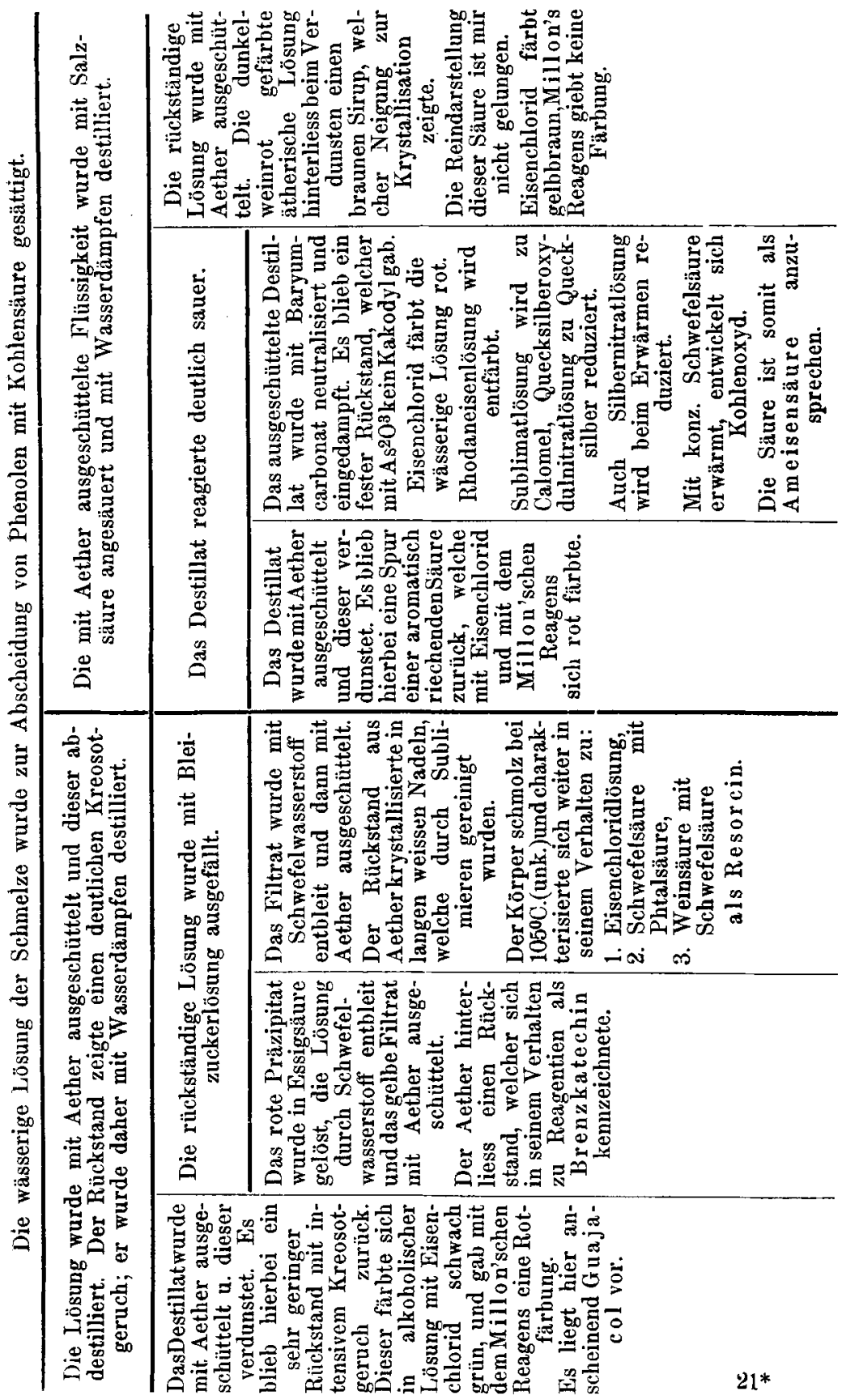


eine $4 \%$ ige Kaliumpermanganatlösung. Hin und wieder wurde etwas verdünnte Schwefelsäure hinzugesetzt, darnit die Flüssigkeit saure Reaktion behielt.

Das Baptigenin löste sich hierbei unter schwacher Kohlensäureentwickelung langsam auf, trotzdem war es unter diesen Umständen der Einwirkung von Permanganat nur schwer zugänglich.

Wenn die Permanganatfarbe längere Zeit bestehen blieb, wurde die Lösung mit etwas schwefliger Säure entërbt, auf dem Wasserbade eingedampft und mit Aether ausgeschüttelt. Beim langsamen Verdunsten der ätherischen Lösung an der Luft blieb nur eine sehr geringe Menge einer nadelförmig-krystallinischen Substanz zurück. Diese Krystalle reagierten sauer und zeigten mit Eisenchlorid eine Rotfärbung.

Der geringen Ausbeute wegen wurde von der Oxydation mit Kaliumpermanganat in saurer Lösung vorläufig Abstand genommen, und wandte ich mich der Oxydation in alkalischer Lösung zu, um hierbei vielleicht eine bessere Ausbeute zu erzielen.

$\mathrm{Zu}$ diesem Zwecke löste ich $4 \mathrm{~g}$ Baptigenin in etwa $200 \mathrm{cc}$ $10 \%$ ige Natronlauge und fügte bei gewöhnlicher Temperatur eine $4 \%$ ige Kaliumpermanganatlösung in kleinen Portionen hinzu, wobei Erwärmung der Flüssigkeit möglichst vermieden wurde. Das Kaliumpermanganat wurde unter Abscheidung von Manganperoxydhydrat rasch reduziert. Es trat hierbei gleich von Anfang an ein piperonalartiger Geruch auf. Mit dem Zusatz von Kaliumpermanganatlösung wurde so lange fortgefahren, bis die grüne Manganatfarbe längere Zeit bestehen blieb. Die Flüssigkeit wurde dann mit verdünnter Schvefelsäure angesäuert und der Braunstein durch Einleiten von schwefliger Säure gelöst. Die überschüssige schweflige Säure wurde durch Einleiten von Kohlensäure entfernt.

Die Lösung zeigte einen starken piperonalähnlichen Geruch. Sie wurde zur Isolierung etwaiger flüchtiger Substanzen aus einer Retorte der Destillation unterworfen. Das Destillat zeigte den nämlichen Geruch und schwach saure Reaktion. Es wurde mit Aether ausgeschüttelt und die ätherische Lösung an der Luft der freiwilligen Verdunstung überlassen. Es blieben hierbei schwach gelblich gefärbte, ölartige Tropfen zurück, welche nach einiger Zeit krystallinisch erstarrten und den piperonalartigen Geruch wie vorher deutlich erkennen liessen.

Die Menge reichte für eine Schmelzpunktbestimmung leider nicht aus. Ich habe mich daher vorläufig auf einige vergleichende Reaktionen. mit dem Piperonal beschränken müssen: 


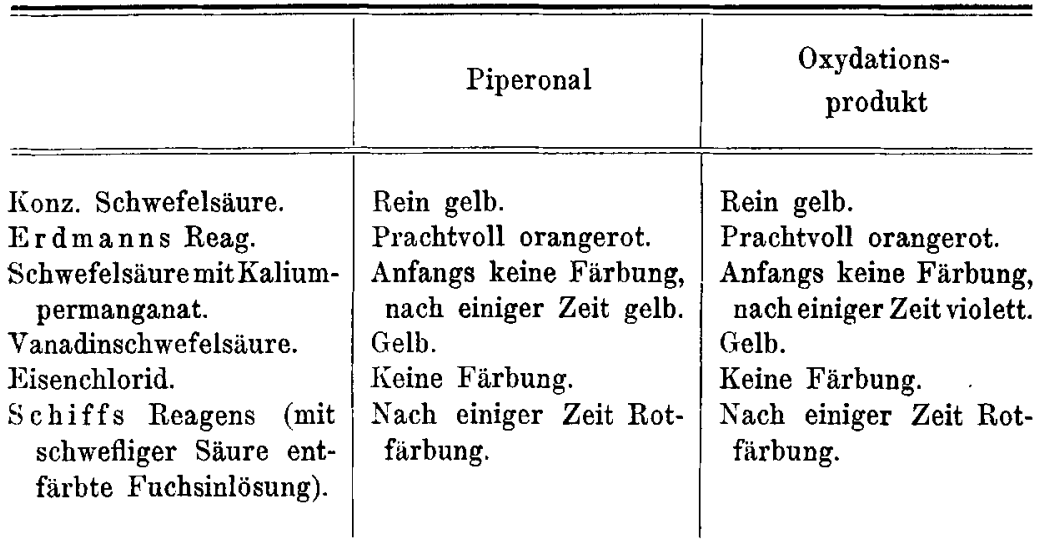

Wie aus obiger Tabelle hervorgeht, ist die Uebereinstimmung beider Körper eine sehr auffällige.

Die Lösung, welche in der Retorte zurückgeblieben war, wurde mit Aether ausgeschüttelt und dieser verdunstet. Es blieben nadelförmige Krystalle zurück, welche teilweise zu Rosetten gruppiert waren and eine gelbliche Farbe zeigten. Sie waren in Wasser, Alkohol und Aether bei gewöhnlicher Temperatur schwer löslich, gaben mit Eisenchlorid keine Färbung und reagierten sauer. Die Substanz wurde aus verdünntem Alkohol umkrystallisiert, war aber auch dann noch schwach gelblich gefärbt und schmolz bei $212-214^{\circ} \mathrm{C}$. (unk.)

Es wurde daher eine Reinigung der Substanz durch Sublimation zwischen zwei Uhrgläsern durch eine Scheibe Filtrierpapier versucht. Hierbei wurde sie in schönen, zarten, blendend weissen Prismen erhalten, welche bei $212-214^{\circ} \mathrm{C}$. (unk.) unter geringer Braunfärbung schmolzen.

Da die Ausbeute viel zu wünschen übrig liess, wurde durch eine geringe Abänderung in dem Verfahren eine bessere Ausbeute zu erzielen versucht.

$4 \mathrm{~g}$ Baptigenin wurden in $300 \mathrm{cc} 5 \%$ Kalilauge gelöst und zu der etwa $30^{\circ} \mathrm{C}$. heissen Flüssigkeit $300 \mathrm{cc} 3 \%$ Kaliumpermanganatlösung unter Umrühren hinzugefügt. Nach dem Absetzen wurde von dem Braunstein abfiltriert und dieser mit heissem Wasser nachgewaschen.

Die Flüssigkeit zeigte nicht vom Anfang an den penetranten, piperonalartigen Geruch, wie bei dem ersten Versuch. Das Filtrat wurde mit verdünnter Schwefelsäure genau neutralisiert und dann aus einer Retorte etwa der dritte Teil abdestilliert. 
Das Destillat wurde mit Aether ausgeschüttelt; die ätherische Lösung hinterliess beim Verdampfen einen weissen festen Rückstand, welcher bei $217-219^{\circ} \mathrm{C}$. (unk.) schmolz.

Der Körper zeigte in alkoholischer Lösung schwach saure Reaktion und gab in dieser Lösung mit Eisenchlorid keine Färbung. Schwefelsäure allein und auch Schwefelsäure mit einer Spur Salpetersäure (Erdmanns Reagens) färbten ihn gelb.

Die Substanz wurde durch Sublimieren zwischen zwei Uhrgläsern in kleinen Nädelchen vom Schmelzpunkt $218-219^{\circ} \mathrm{C}$. (unk.) erhalten.

Offenbar war unter diesen Versuchsbedingungen ein ganz andrer Körper entstanden, wie bei dem ersten Oxydationsversuch.

Die rückständige Lösung in der Retorte wurde auf dem Wasserbade eingedampft. Durch Zusatz von verdünnter Schwefelsäure entstand ein Niederschlag, welcher aus verdünntem Alkohol umkrystallisiert wurde. (Ausbeute $70 \mathrm{mg}$.).

Der Schmelzpunkt wurde bei $225^{\circ}$ C. (unk.) gefunden.

Die Substanz wurde sublimiert und schmolz dann bei 209 bis $214^{\circ}$ C. (unk.)

Einwirkung von Natronlauge auf das Baptigenin.

Wie ich schon früher bei der Acetylbestimmung des Triacetylbaptigenins erörtert habe, entsteht bei der Einwirkung von Natronlauge auf diese Substanz ein neuer Körper, welcher in silberglänzenden Blättchen krystallisiert und bei $148^{\circ} \mathrm{C}$. (unk.) schmilzt. Ich habe versucht, die gleiche Substanz durch Einwirkung von Natronlauge auf das Baptigenin, bez. auf das Baptisin zu gewinnen.

$1 \mathrm{~g}$ Baptigenin wurde zu diesem Zwecke mit 5\% Natronlauge auf der Asbestpappe am Rückflusskühler erhitzt. Es löste sich die Substanz anfangs mit grüner Farbe, welche sich bald in eine rotbraune umwandelte. Nach zweistïndigem Kochen wurde die Lösung, welche einen aromatischen Geruch zeigte, mit Salzsäure angesäuert. Es schied sich hierbei eine harzartige Masse aus. Diese wurde nach dem Abkühlen gesammelt, in verdünntem Alkohol gelöst und mit frisch ausgeglühter Tierkohle möglichst entfärbt. Aus dem Filtrat schied sich der neue Körper krystallinisch aus; durch mehrmaliges Umkrystallisieren aus verdünntem Alkohol konnte er schliesslich in silberglänzenden Blättchen vom Schmelzpunkt $148^{\circ} \mathrm{C}$. (unk.) erhalten werden.

Da das Entfärben bei dieser Darstellung eine sehr zeitraubende und langwierige Arbeit ist, habe ich mich nach einer bequemeren Beseitigung der harzartigen Produkte umgesehen und diese auch mit bestem Erfolge durchführen können, wenn ich die harzartige Masse mit 
Ligroin auskochte. Das Ligroin lässt den Farbstoff vollständig ungelöst zurück, so dass beim Abkühlen der Ligroinlösung der neue Körper in weissen Nadeln direkt auskrystallisiert.

Für diesen Zweck eignet sich auch das Benzol ganz gut. Man erhält hierbei den Körper zwar etwas mehr gefärbt, jedoch ist es unschwer ihn durch mehrmaliges Umkrystallisieren aus verdünntem Alkohol rein zu erhalten.

Für die Darstellung in etwas grösseren Mengen dieser Verbindung, die als Baptigenetin bezeichnet sein mag, habe ich später das Baptisin direkt als Ausgangsmaterial gewählt.

Bei der Einwirkung von Natronlauge auf das Baptigenin, bezw. Baptisin entstehen auch Spuren einer aromatisch (piperonalähnlich) riechenden Substanz, welche durch Destillieren der alkalischen Flüssigkeit und Ausschütteln des Destillates mit Aether gewonnen werden können.

\section{Baptigenetin: $\mathrm{C}^{12} \mathrm{H}^{10} \mathrm{O}^{4}$.}

Das Baptigenetin krystallisiert aus verdünntem Alkohol in farblosen, silberglänzenden Blättchen, vom Schmelzpunkt $148^{\circ} \mathrm{C}$. (unk.) Es löst sich in kaltem Wasser nur ganz wenig, etwas mehr in heissem. Mit Wasserdämpfen ist es nicht flüchtig. In heissem verdünntem Alkohol ist es leicht, in kaltem wenig löslich. Es löst sich leicht in 95\% Weingeist, sowie in Chloroform, Aceton, Aether, Essigäther und Eisessig. In Schwefelkohlenstoff ist das Baptigenetin unlöslich. In heissem Ligroin löst es sich ziemlich gut auf und krystallisiert beim Abkühlen vollständig wieder aus. Benzol löst es in der Siedehitze leichter als Ligroin.

In alkoholischer Lösung reagiert das Baptigenetin sehr schwach sauer. Eisenchlorid färbt diese Lösung, ebenso wie die Lösung des Baptigenetins in Aceton, intensiv rot. Diese Färbung verschwindet durch Salzsäure nicht; Zusatz von Sodalösung giebt keinen charakteristischen Farbenumschlag (das Eisen wird gefällt). Schwefelsäure allein färbt anfangs schmutzig olivengrün, später violett.

Schwefelsäure mit einer Spur Salpetersäure (Erdmanns Reagens) färbt intensiv rotbraun.

Mit Natriumhypochlorit giebt das Baptigenetin eine Rotfärbung. Es reduziert die Fehling'sche Kupferlösung beim Kochen nicht, wohl aber beim Stehen in der Kälte die ammoniakalische Silberlösung.

Durch Lösen molekularer Mengen von Baptigenetin und Pikrinsäure in Aceton, und Verdunsten des Lösungsmittels bei gewöhnlicher 
Temperatur, resultieren nur Einzelkrystalle von Baptigenetin und von Pikrinsäure. Das Baptigenetin bildet somit keine Doppelverbindung mit Pikrinsäure.

Das Baptigenetin ist in $4 \%$ Lösung in Aceton optisch inaktiv.

Es schmilzt ohne zu sublimieren; die geschmolzene Masse erstarrt wieder krystallinisch.

Die bei $100^{\circ} \mathrm{C}$. getrocknete Substanz gab folgende analytische Daten.

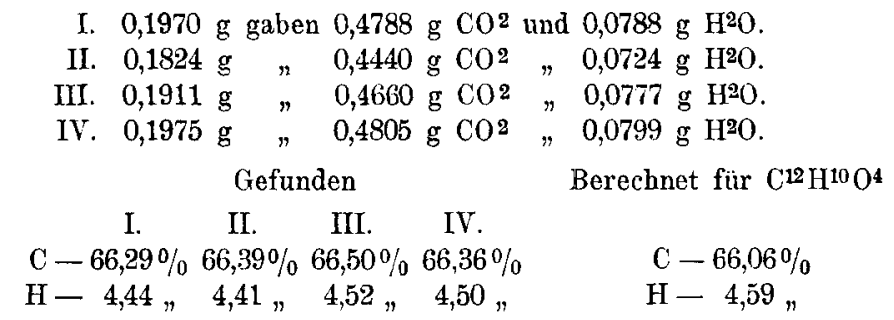

Die Formel des Baptigenetins $\mathrm{C}^{12} \mathrm{H}^{10} \mathrm{O}^{4}$ differiert somit um $\mathrm{C}^{2} \mathrm{H}^{2} \mathrm{O}^{2}$ von der des Baptigenins $\mathrm{C}^{14} \mathrm{H}^{12} \mathrm{O}^{6}$.

Ich habe mich zunächst bemüht, Aufschluss über die Anzahl der Hydroxylgruppen, welche noch in dem Baptigenetin vorhanden sind, zu erhalten.

\section{Diacetylanhydrobaptigenetin: $\mathrm{C}^{12} \mathrm{H}^{6} \mathrm{O}^{3}\left(\mathrm{C}^{2} \mathrm{H}^{3} \mathrm{O}\right)^{2}$.}

Für die Acetylierung wurde $1 \mathrm{~g}$ Baptigenetin mit $1 \mathrm{~g}$ frisch entwässertem essigsaurem Natrium und $10 \mathrm{~g}$ Essigsäureanhydrid während zweier Stunden am Rückflusskühler gekocht, wobei die Substanz sich leicht zu einer gelblichroten Flüssigkeit löste. Diese wurde dann mit Wasser verdünnt und die abgeschiedene, braungefärbte, krystallinische Masse mit etwas absolutem Alkohol ausgewaschen. Hierbei blieb eine weisse, krystallinische Substanz ungelöst zurück, welche sich in Aceton leicht löste.

Beim freiwilligen Verdunsten der Acetonlösung an der Luft krystallisierte der neue Körper in tafelförmigen, gut ausgebildeten Krystallen. Diese verloren beim Trocknen bei $100^{\circ} \mathrm{C}$. nichts an Gewicht. Der Schmelzpunkt wurde bei $192--194^{\circ}$ C. (unk.) gefunden. Eisenchlorid färbte die Acetonlösung nicht rot.

Eine Elementaranalyse gab folgende Daten:

$0,2031 \mathrm{~g}$ gaben $0,5030 \mathrm{~g} \mathrm{CO} \mathrm{CO}^{2}$ und $0,0779 \mathrm{~g} \mathrm{H}^{2} \mathrm{O}$.

$\begin{array}{cc}\text { Gefunden } & \text { Berechuret für } \mathrm{C}^{12} \mathrm{H}^{6} \mathrm{O}^{3}\left(\mathrm{C}^{2} \mathrm{H}^{3} \mathrm{O}\right)^{2} \\ \mathrm{C}-67,54^{0} & \mathrm{C}-67,60^{0} \\ \mathrm{H}-4,26 \% & \mathrm{H}-4,22 \%\end{array}$


Es ist also bei der Acetylierung des Baptigenetins unter gleichzeitiger Wasserabspaltung ein Diacetylanhydrobaptigenetin entstanden.

Nach den vorliegenden Beobachtungen über das Baptigenetin, im Zusammenhang mit der Thatsache, dass das Baptisin bei der Kalischmelze im wesentlichen Resorcin und Brenzkatechin nebeneinander lieferte, lag die Vermutung nahe, dass dieser Körper ein Tetraoxydiphenyl von folgender Struktur sei.

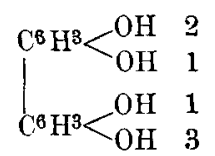

Mit dieser Formel würde die gleichzeitige Bildung von Resorcin und Brenzkatechin bei der Kalischmelze im vollen Einklang stehen. In diesem Falle muisste jedoch das Baptigenetin bei der Zinkstaubdestillation Diphenyl liefern, was jedoch, wie die bezüglichen Versuche lehrten, sich nicht als zutreffend erwies. In welcher Beziehung Baptigenin und Baptigenetin $\mathrm{zu}$ einander stehen, habe ich vorläufig noch nicht entscheiden können. Ich habe mich jedoch überzeugt, dass das Baptigenetin aus dem Baptigenin unter gleichzeitiger Abspaltung von Ameisensäure hervorgeht.

\section{Physiologische Wirkung des Baptisins.}

Um zu konstatieren, ob das Baptisin giftig oder ungiftig sei, wurden einige Versuche an Landfröschen (Rana temporaria) angestellt. Weil das Glykosid in Wasser sich nicht löst, wurde es mit Wasser gut zerrieben und dann in dieser Form subcutan eingespritzt.

Ich fand hierbei, dass $10 \mathrm{mg}$ selbst nach längerer Zeit keine giftige Wirkung äusserten. Es wurde dann noch zweimal jedesmal $10 \mathrm{mg}$ bei demselben Frosch eingespritzt; jedoch das Tier blieb ganz normal wie vorher.

Auch Versuche, wobei ich $50 \mathrm{mg}$ Baptisin auf einmal einspritzte, hatten denselben negativen Erfolg. Es ist das Baptisin somit für Frösche vollständig ungiftig.

\section{B. Baptitoxin,}

das Alkaloid der Wurzel von Baptisia tinctoria R.Br.

Darstellung. Der verdünnt alkoholische Auszug der Wurzel enthielt, wie schon früher erörtert, nur Spuren von Alkaloid. Die mit Alkohol erschöpfte Wurzel wurde daher noch mit salzsäurehaltigem Wasser in der Wärme extrahiert, die so erhaltene Flüssigkeit im Wasserbade eingedampft und mit Natronlauge bis zur stark alkalischen Reaktion versetzt. Aus dieser Lösung wurde das Alkaloid durch Chloroform ausgeschüttelt, das Chloroform abdestilliert und der rückständige, schwach braun gefärbte Sirup in absolutem 
Alkohol gelöst. Diese Lösung reagierte stark alkalisch auf Lackmus. Nach Zusatz von $50 \%$ Salpetersäure bis zur schwach sauren Reaktion krystallisierte das salpetersaure Salz in schönen, öfters zu Rosetten gruppierten, weissen Blättchen aus. Die Krystalle wurden gesammelt, mit wenig absolutem Alkohol nachgewaschen und getrocknet.

Die Ausbeute aus $4,1 \mathrm{~kg}$ der Wurzel betrug nur $1,2 \mathrm{~g}$ salpetersaures Salz. Es wurde in etwas Wasser gelöst und diese Lösung über Schwefelsäure der Krystallisation überlassen.

\section{Baptitoxinnitrat. (Cytisinnitrat.)}

Das salpetersaure Salz wurde aus Wasser in grossen, harten, gut ausgebildeten Krystallen erhalten, welche noch eine schwach gelbliche Farbe zeigten. Es wurde zwischen Fliesspapier getrocknet, zerrieben und dann bei $100^{\circ} \mathrm{C}$. bis zum konstanten Gewicht erhitzt.

$0,4272 \mathrm{~g}$ verloren dabei $0,0278 \mathrm{~g}$ Wasser.

$\begin{array}{cc}\text { Gefunden } & \text { Berechnet für } \mathrm{C}^{11} \mathrm{H}^{14} \mathrm{~N}^{2} \mathrm{O} \cdot \mathrm{HNO}^{3}+\mathrm{H}^{2} \mathrm{O} \\ \mathrm{H}^{2} \mathrm{O}-6,51 \% & \mathrm{H}^{2} \mathrm{O}-6,64 \% .\end{array}$

Das wasserfreie Salz wurde zur Bestimmung der spezifischen Drehung angewendet. Dieselbe ergab sich als:

$$
[\alpha] \mathrm{D}=-85^{\circ} 52^{\prime} \quad \begin{array}{ll}
\mathrm{l}=2 & \mathrm{~d}=1,01 \\
\mathrm{p}=2,873 & \mathrm{t}=19^{\circ} \mathrm{C} .
\end{array}
$$

Van de Moer ${ }^{1}$ ) fand für das Cytisinnitrat in wässriger Lösung:

$$
\begin{array}{lll}
{[\alpha] \mathrm{D}=-90^{\circ} 10^{\prime},} & \mathrm{c}=5, & \mathrm{l}=2, \quad \mathrm{t}=11^{\circ} \mathrm{C} . \\
{[\alpha] \mathrm{D}=-89^{\circ} 20^{\prime},} & \mathrm{c}=2,5 & \mathrm{l}=2, \quad \mathrm{t}=11^{\circ} \mathrm{C} .
\end{array}
$$

Für das krystallisierte Cytisinnitrat in wässeriger Lösung giebt. Partheil ${ }^{2}$ ) an:

$$
\begin{gathered}
{[a] D=-82^{\circ} 37^{\circ} \quad p=1,9848 ; d=1,0075 ; t=17^{\circ} \mathrm{C} .} \\
\text { Baptitoxin. } \\
\text { (Cytisin.) }
\end{gathered}
$$

Die freie Base schmolz bei $152-153^{\circ} \mathrm{C}$. (unk.), übereinstimmend mit den Angaben von Partheil ${ }^{3}$ ).

Sie löste sich leicht in Wasser; diese wässerige Lösung reagierte stark alkalisch auf Lackmus. Die Base erwies sich auch als leicht löslich in Alkohol, Chloroform und Essigäther.

1) Arch. d. Pharm. 1891. 40.

2) Arch. d. Pharm. 1892. 460.

3) Arch. d. Pharm. 1892. 458. 
Eisenchlorid gab eine Rotfärbung, welche durch eine Spur Wasserstoffsuperoxydlösung verschwand. Beim gelinden Erwärmen trat eine schön blaue Färbung auf: Van de Moer'schen Cytisinreaktion.

Die spezifische Drehung des Baptitoxins wurde für eine wässerige Lösung desselben von $0,4220 \mathrm{~g}$ in $14,2296 \mathrm{~g}$ Wasser im I uaurent'schen Halbschattenapparat bestimmt.

Ich fand dabei $[\alpha] \mathrm{D}=-118^{\circ} 55^{\triangleleft} \quad \mathrm{p}=2,88 ; \mathrm{d}=1,01 ; \mathrm{l}=2 ; \mathrm{t}=190 \mathrm{C}$.

Van de Moer ${ }^{1}$ ) fand für die wässerige Cytisinlösung:

$$
[\alpha]_{D}=-120^{\circ} \quad c=2 ; 1=2 ; t=12^{\circ} \mathrm{C} \text {. }
$$

Partheil ${ }^{2}$ ) erhielt folgendes Resultat:

$$
[c .]_{D}=-119^{0} 57^{\prime} \quad \mathrm{p}=1,9908 ; \mathrm{d}=1,0046 ; \mathrm{t}=17^{\circ} \mathrm{C} .
$$

Baptitoxinchlorhydrat.

(Cytisinchlorhydrat.)

Das Salz wurde aus Wasser in grossen Krystallen erhalten.

Eine Krystallwasserbestimmung des zwischen Fliesspapier getrockneten Salzes gab folgendes Resultat:

$0,3730 \mathrm{~g}$ verloren beim Trocknen beì $100^{\circ} \mathrm{C} .0,0280 \mathrm{~g}$ Wasser.

$\begin{array}{cc}\text { Gefunden } & \text { Berechnet für } \mathrm{C}^{11} \mathrm{H}^{44} \mathrm{~N}^{2} \mathrm{O} \cdot \mathrm{HCl}+\mathrm{H}^{2} \mathrm{O} \\ \mathrm{H}^{2} \mathrm{O}-7,50 \% & \mathrm{H}^{2} \mathrm{O}-7,36 \%\end{array}$

Von diesem bei $100^{\circ} \mathrm{C}$. getrockneten Salze wurden zwei Salzsäurebestimmungen ausgeführt:

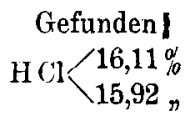

Berechnet für $\mathrm{C}^{11} \mathrm{H}^{14} \mathrm{~N}^{2} \mathrm{O} \cdot \mathrm{HCl}$

$$
\mathrm{HCl} \cdot 16,11 \%
$$

Aus dem Filtrat von dieser Salzsäurebestimmung wurde das Goldsalz dargestellt.

Baptitoxingoldchlorid (Cytisingoldchorid): $\mathrm{C}^{11} \mathrm{H}^{14} \mathrm{~N}^{2} \mathrm{O} \cdot \mathrm{HAu} \mathrm{Cl}^{4}$.

Das Baptitoxingoldchlorid wird aus der mit Salzsäure angesäuerten Lösung als ein zitronengelber Niederschlag gefällt. Aus salzsäurehaltigem Wasser umkrystallisiert, stellt es rotbraune, meistens hakig gekrümmte Krystallnädelchen dar vom Schmelzpunkt $212^{0}$ bis $213^{\circ}$ C. (unk.) wie auch Partheil ${ }^{B}$ ) für das Cytisingoldchlorid angiebt. Es enthält kein Krystallwasser.

1) Arch. d. Pharm. 1891. 40.

2) Arch. d. Pharm. 1892. 459.

3) Arch. d. Pharm. 1892. 469. 
Eine Verbrennung im Bleichromatrohr mit vorgelegter reduzierter Kupferspirale gab folgendes Resultat:

$0,2379 \mathrm{~g}$ Goldsalz gaben $0,2155 \mathrm{~g} \mathrm{CO}^{2}$ und $0,0704 \mathrm{~g} \mathrm{H}_{2} \mathrm{O}$. Im Schiffchen blieben $0,0888 \mathrm{~g}$ Gold zurück.

$\begin{array}{cr}\text { Gefunden } & \text { Berechnet für } \mathrm{C}^{11} \mathrm{H}^{14} \mathrm{~N} 2 \\ \mathrm{C}-24,70 \% & \mathrm{C}-24,92 \% \\ \mathrm{H}-3,28 \% & \mathrm{H}-2,83 \% \\ \mathrm{Au}-37,32, & \mathrm{Au}-37,12,\end{array}$

Die völlige Uebereinstimmung des Baptitoxins mit dem Cytisin ergab sich weiter auch durch die physiologische Wirkung desselben. Die Wirkung beider Basen auf Frösche war durchans die gleiche.

Vorliegende Arbeit wurde auf Veranlassung des Herrn Prof. Dr. P. C. Plugge im pharmazeutisch-chemischen Laboratorium in Groningen angefangen und unter Leitung des Herm Geheimrat Prof. Dr. E. Schmidt im pharmazentisch-chemischen Institut in Marburg fortgesetzt und abgeschlossen.

Die weiteren Untersuchungen über die Bestandteile der Wurzel von Baptisia tinctoria R.Br. werden vorbehalten.

\section{Ueber das Carpain.}

\section{Mitteilung von Dr. J. J. L. van Rijn. ${ }^{1}$ )}

(Eingegangen den 3. Mai 1897).

Nach meiner ersten Publikation über dieses Alkaloid habe ich mich sehr darum bemüht, um mehr Material zur Fortsetzung meiner Untersuchungen $\mathrm{zu}$ erhalten. $\mathrm{Da}$ die verschiedenen Handelsfirmen noch immer $30 \mathrm{Mk}$. für das Gramm Carpaïn notieren, konnte ich nicht daran denken, dasselbe aus einer dieser Quellen zu beziehen und entschloss ich mich daher, Rohmaterial aus Indien kommen zu lassen, um das Alkaloid selbst darzustellen. Es ist mir gelungen, ungefähr $500 \mathrm{~kg}$ getrocknete Blätter der Carica Papaya $L$ zu erhalten, welche teilweise in früher von mir beschriebener Weise auf Alkaloid verarbeitet wurden. Leider war aber der Alkaloidgehalt ziemlich gering, so dass es geraume

1) I. Mitteilung diese Zeitschrift 1893, S. 184. 